\title{
QTL Mapping And Candidate Gene Mining of Flag Leaf Size Traits In Japonica Rice Based On Linkage Mapping And Genome-Wide Association Study
}

\author{
Wang Jiangxu \\ HAAS: Heilongjiang Academy of Agricultural Sciences \\ Wang Tao \\ HAAS: Heilongjiang Academy of Agricultural Sciences \\ Wang qi \\ HAAS: Heilongjiang Academy of Agricultural Sciences
}

\section{Tang Xiaodong}

HAAS: Heilongjiang Academy of Agricultural Sciences

\section{Ren Yang}

HAAS: Heilongjiang Academy of Agricultural Sciences

Zheng Haiyan

HAAS: Heilongjiang Academy of Agricultural Sciences

\section{Liu Kai}

HAAS: Heilongjiang Academy of Agricultural Sciences

\section{Yang Luomiao}

NEAU: Northeast Agricultural University

Jiang Hui

HAAS: Heilongjiang Academy of Agricultural Sciences

\section{Li Yidan}

HAAS: Heilongjiang Academy of Agricultural Sciences

Liu qi

HAAS: Heilongjiang Academy of Agricultural Sciences

\section{Zou Detang}

NEAU: Northeast Agricultural University

Zheng Hongliang ( $\nabla$ hongliangzheng@neau.edu.cn )

HAAS: Heilongjiang Academy of Agricultural Sciences

\section{Research Article}

Keywords: Japonica rice, QTLs, Flag Leaf, Linkage mapping, Genome-wide association study

Posted Date: September 16th, 2021

DOI: https://doi.org/10.21203/rs.3.rs-866138/v1 
License: (a) (1) This work is licensed under a Creative Commons Attribution 4.0 International License. Read Full License 


\section{Abstract}

As one of the most important part of the ideal plant type of japonica rice, leaf shape affects the photosynthesis and carbohydrate accumulation directly. Mining and using new leaf shape related genes/QTLs can further enrich the theory of molecular breeding and accelerate the breeding process of japonica rice. In the present study, 2 RILs and a natural population with 295 japonica rice varieties were used to map QTLs for flag leaf length (FL), flag leaf width (FW) and flag leaf area (FLA) by linkage analysis and genome-wide association study (GWAS) through 2 years. A total of 64 QTLs were detected by 2 ways, and pleiotropic QTLs qFL2 (Chr2_33,332,579) and qFL 10 (Chr10_10,107,835; Chr10_10,230,100) consisted of overlapping QTLs mapped by linkage analysis and GWAS through 2 years were identified. The candidate genes LOC_Os02g54254, LOC_Os02g54550, LOC_Os10g20160, LOC_Os10g20240, LOC_Os10g20260 were obtained, filtered by linkage disequilibrium (LD), and haplotype analysis. LOC_Os10g20160 (SD-RLK-45) showed outstanding characteristics in quantitative real-time PCR (qRT-PCR) analysis in leaf development period, belongs to S-domain receptor-like protein kinases gene and probably to be a main gene regulating flag leaf width of japonica rice. The results of this study provide valuable resources for mining the main genes/QTLs of japonica rice leaf development and molecular breeding of japonica rice ideal leaf shape.

\section{Introduction}

Rice is one of the most important food crops on the planet, bearing the lifeline of human food security (Pane et al 2021; Barboza et al 2018; Timmer 2014). Leaf is an important part of rice plant histogenesis and morphogenesis, and also the main organ for photosynthesis and respiration (Adachi et al 2014; Zhu et al 2014; Gu et al 2012). The photosynthetic energy storage and normal life function of rice are directly affected by leaf (He et al 2017). Meanwhile, rice leaf size is an important part of rice ideal plant type, as well as an important character of rice yield formation (Rahman et al. 2013). To be the top functional leaf of rice, flag leaf has significant effects on plant related physiological characters and field population structure (Zhu et al 2012; Giuliani et al. 2013). Therefore, it is an effective way to improve the quality and yield of rice by shaping and screening the leaf morphology in the process of breeding.

Although leaf size has a great influence on high photosynthetic efficiency, the genetic mechanism of leaf morphological characteristics is still unclear (Hoang et al 2019). Mining QTLs by linkage mapping in RILs and GWAS mapping in natural populations to find the candidate genes might be the most efficient way to analyze the genetic basis of rice leaf shape.

In recent years, many QTLs and regulatory genes related to rice leaf morphology have been discovered (http://www.gramene.org/) (Fujino et al. 2008; Farooq et al. 2010; Chen et al. 2012; Zhang et al. 2014). These QTLs and genes can change the physiological function of plants by regulating leaf morphology, and have important impacts on the coordination of light energy utilization and "sink-source" relationship. At the same time, their potential impact on rice yield has also been gradually discovered. Tang et al. (2018) Using CSSL population with 143 individuals, and obtained 14 leaf length and 19 leaf width QTLs, and further obtained the rice leaf size gene Ghd7.1 by fine mapping, mutation test, and allelic variation analysis. Zhang et al. (2015a) mapped the flag leaf width QTL qflw7.2 to $27.1 \mathrm{~kb}$ by recombinant inbred lines derived from $93-11$ and Peiai 64s, and has identified 2 candidate genes LOC_Os07g41180, LOC_Os07g41200. A natural population of 532 individuals was used for genome-wide association analysis and high-throughput leaf scoring, 73 QTLs associated with rice leaves were mapped as a result (Yang et al. 2015). At present, we've got a clear understanding of the cloned gene NAL 1 located on chromosome 4, which affects the growth of lateral leaf (Jiang et al. 2015). The NAL 1 mutant is characterized by dwarf and narrow 
leaf, and the expression level of the genes related to the polar transport of auxin and leaf development in the mutant changed ( $\mathrm{Li}$ et al. 2019a). It was highly expressed in vascular tissue, which played an important role in cell division and cell size regulation, promoting the lateral growth of leave. OsFLW7 regulated the width of flag leaf, increased photosynthetic leaf area (Xu et al., 2017). At the same time, OsFLW7 is an allele of GL7/ GW7, which may be related to the regulation of grain traits and the mutant was found that the grain length, grain plumpness and yield increased significantly.

GWAS and linkage analysis are both accurate and effective tools for QTL detection of complex rice traits (Famoso et al. 2011). The breadth and accuracy of QTL detection could be significantly improved by combining these 2 methods. In this study, 2 sets of RILs populations with high-density bin-map and 295 re-sequenced japonica rice accessions were used to conducted linkage/GWAS mapping of flag leaf length, width and area of japonica rice. Two novel QTL qFL2, FL10 and five candidate genes related to flag leaf size in japonica rice were discovered, providing important references for molecular breeding of japonica rice with ideal leaf size and plant type.

\section{Methods}

\section{Populations for QTL Mapping}

Natural population is composed of 295 rice varieties, most of which are temperate japonica rice, widely collected from Heilongjiang, Jilin and Liaoning provinces, while foreign materials mainly come from Japan, Korea, the Democratic People's Republic of Korea and Russia. This population has been used in previous studies (Li et al. 2019b). RILsA contains 195 individuals, obtained from crossing the narrow erect leaf japonica rice variety K131 and wide curved leaf upland rice variety HDB. RILsB contains 189 individuals, which was derived from a cross between the long wide flag leaf japonica rice variety WD20342 and short narrow flag leaf variety Caidao. All materials were planted in Acheng rice experimental base of Northeast Agricultural University from 2019 to 2020. Each individual was planted in 8 rows with 20 plants in each row by single plant transplanting and the spacing of rows and plants was $30 \mathrm{~cm} \times 3 \mathrm{~cm}$. The field management of water and fertilizer followed the basic method of conventional field production.

\section{Phenotypic Identification of Flag Leaf Size}

In order to reduce the influence of marginal effect on phenotype, five continuous plants in the middle of the fourth row of each variety were selected as the research objects, and the average value of five plants for each line was calculated. The flag leaf length (FL), width (FW) and area (FLA) were investigated at full heading stage using Tuopu YMJ-D Living Leaf Area Meter (Tuopu Yunnong Technology Co., Ltd). Population phenotypic correlation analysis for QTL mapping was performed by SPSS.

\section{Linkage Analysis and Genome-wide Association Study}

Two linkage maps for linkage analysis were constructed through 10K array genotyping by targeted sequencing (GBTS) supported by MOLBREEDING Biotechnology Co., Ltd (Shijiazhuang, China). The RILsA population has been used in previous study ( $\mathrm{Li}$ et al. 2020). Nine hundred and seventy-eight bin markers covered $2465.32 \mathrm{cM}$ of the rice genome with an average distance of $2.52 \mathrm{cM}$ constructed the linkage map (Fig. S1). The linkage map of RILsB contains 527 bin markers covered $1874.85 \mathrm{cM}$ of the rice genome with an average distance of $3.56 \mathrm{cM}$ (Fig. S2). The IciMapping Ver.4.2 (Meng et al. 2015) based on inclusive composite interval mapping (ICIM) was used to detected the QTLs for rice flag leaf traits. The walking speed was set as $1 \mathrm{~cm}$, and the LOD threshold of ICIM was set as 2.5 . To ensure the accuracy of mapping results, we controlled the type 1 error of whole genome detection below $5 \%$ by 
1000 permutation tests. The natural population for genome-wide association study (GWAS) was deep re-sequenced by Beijing Genomics Institute (BGI www.genomics.org.cn). A total of 788, 396 SNPs meeting the criteria (minimum allele frequency $\geq 5 \%$, deletion rate $\leq 20 \%$ ) were selected for follow-up analysis, and 295 japonica rice varieties' population structure analysis, genetic relationship analysis and linkage disequilibrium analysis have been completed in the previous study of our laboratory (Li et al. 2019b). The mixed linear model (MLM) of TASSEL 5.0 (Bradbury et al. 2007) was used for genome-wide association analysis and setting the threshold of SNPs significantly associated with flag leaf traits as $5.46 \times 10^{-6}$ which was calculated by GEC software (http://statgenpro.psychiatry.hku.hk/gec/). If 2 or more SNPs were located in the same LD interval, they were regarded as the same QTL, and the SNP with the smallest $\mathrm{p}$ value was treated as the lead SNP. QQman package in R was used to create the Manhattan and Q-Q plots (Turner 2014).

\section{Haplotype Analysis of Candidate Genes}

Considering the LD decay of the whole genome was confirmed in previous study by $109 \mathrm{~kb}$ (Li et al. 2019b), we selected $218 \mathrm{~kb}$ upstream and downstream of the SNP as the target interval to screen candidate genes. Nonsynonymous SNPs of all exons were extracted from the "Rice SNP-Seek Database" of The International Rice Informatics Consortium (IRIC) (https: //snp-seek.irri.org/), which were then used for haplotype analysis of candidate genes with DnaSP software (Julio et al. 2017).

\section{RNA Extraction and qRT-PCR Analysis}

The flag leaves of four parents (K131, HDB, WD20342, Caidao) of RILs populations took 4-6 days to fully extended. Five repetitions of flag leaves for each parent were sampled 4 times from the flag leaves begin to drew out to fully extended. The total RNA was extracted with TranZol Up RNA Kit (TransGen Biotech). HiFiScript cDNA synthesis kit (CoWin Biosciences, Beijing, China) was used to synthesize cDNA. qRT-PCR was performed on Bio-Rad CFX96 system using 2xFast qPCR Master Mixture with 3 biological replicates for each sample. House-keeping gene Actin 1 was used to measure the mRNA levels of candidate genes ( $\mathrm{Li}$ et al. 2019b) as an internal control. The primers used for qRT-PCR in this study are all shown in Table S1. Relative gene expression levels were determined using the $2^{-\triangle \Delta C t}$ method (Livak and Schmittgen 2001). Data shown in figures and tables are mean values of three replicates.

\section{Results}

\section{Phenotypic Analysis}

The phenotypic data of flag leaf size of RILs populations and natural population from 2019 to 2020 are shown in Table S2; Table S3, and the general trends in 2 years were basically the same. The 3 leaf size traits of parents of RILs populations showed great phenotypic differences in 2 years and the RILs showed significant variation in FL, FW and FLA. FL and FLA in 2 RILs populations with standard deviation from 4.38 to 7.25, presented stronger variation than FW with the standard deviation from $0.17-1.44$. The variation characteristics of flag leaf phenotypic in natural population were similar to those of RILs in 2 years. Most of the absolute values of kurtosis and skewness were near 1 , which basically conformed to the normal distribution and showed a typical genetic model of quantitative traits, was suitable for linkage analysis and genome-wide association analysis.

\section{Linkage Mapping for Flag Leaf Size in Japonica Rice}


We conducted QTL linkage analysis for FL, FW and FLA of 2 RILs populations in 2019 and 2020. A total of 28 QTLs were detected, which were distributed on chromosome 1, 2, 3, 4, 6, 7, 10 and 11 of rice (Table S4). The phenotypic variation explained by a single QTL ranged from 4.97-20.88\%. qFLr7-2 and qFWr2-3 in RILsA; qFLr3, qFWr2-1, qFLAr4-2 and qFWr10 in RILsB were detected in 2 years simultaneously, represents the stable expression of genetic effects in the corresponding interval. At the same time, qFLr6-1, qFLAr6-1in RILsA and qFLr3, qFLAr3-2 in RILsB located in the same interval respectively, but detected to control different traits in different populations and years, which were likely to be pleiotropic QTLs.

\section{GWAS for Flag Leaf Size in Japonica Rice}

A natural population which has been deeply re-sequenced and its 788, 396 high quality SNP markers were used to conducted GWAS. Manhattan and Q-Q plots for the GWAS are shown in Fig. 1, Fig. 2. Table 1, Table 2 show that a total of 36 SNPs were detected under the threshold of $5.46 \times 10^{-6}$ which were significantly associated with flag leaf size of japonica rice. These SNPs were distributed on all chromosomes of rice except chromosome 11 with the $\mathrm{R}^{2}$ ranged from 8.87-12.81\%. The GWAS results showed that Chr10_10,230,100 (qFWn10-2), Chr10_10,107,835 ( $q F L A n 10-1)$ located in one LD interval was detected in both years associated with FW and FLA separately. Chr2_33,332,579 (qFWn2-2, qFLAn2-5) and Chr7_20,475,568 (qFWn7, qFLAn7-2) were detected in 2019 associated with FW and FLA simultaneously. The above-mentioned results are consistent with what we obtained in phenotypic analysis.

Table 1

Significant SNPs associated with flag leaf size related traits in natural population in 2019

\begin{tabular}{|llllllll|}
\hline Traits & QTLs & Peak SNPs & Chr. & Position & Pvalue & $R^{2}(\%)$ & known genes \\
\hline FL & qFLn1 & Chr1_3505761 & 1 & 3505761 & $3.79 \mathrm{E}-06$ & 8.87 & \\
\hline FL & qFLn10 & Chr10_15440761 & 10 & 15440761 & $6.35 \mathrm{E}-07$ & 10.02 & \\
\hline FW & qFWn1-2 & Chr1_18340871 & 1 & 18340871 & $1.88 \mathrm{E}-06$ & 9.37 & \\
\hline FW & qFWn2-2 & Chr2_33332579 & 2 & 33332579 & $3.18 \mathrm{E}-08$ & 12.45 & \\
\hline FW & FFWn3 & Chr3_18626 & 3 & 18626 & $5.19 \mathrm{E}-07$ & 10.38 & \\
\hline FW & qFWn7 & Chr7_20475568 & 7 & 20475568 & $4.39 \mathrm{E}-06$ & 12.81 & OsFLW7 \\
\hline FW & qFWn8 & Chr8_5133437 & 8 & 5133437 & $2.15 \mathrm{E}-07$ & 10.04 & OsBAK1 \\
\hline FW & qFWn10-2 & Chr10_10230100 & 10 & 10230100 & $1.67 \mathrm{E}-06$ & 10.32 & \\
\hline FLA & qFLAn2-2 & Chr2_24488823 & 2 & 24488823 & $4.21 \mathrm{E}-06$ & 8.84 & \\
\hline FLA & qFLAn2-3 & Chr2_31463696 & 2 & 31463696 & $1.28 \mathrm{E}-06$ & 9.60 & \\
\hline FLA & qFLAn2-4 & Chr2_32571605 & 2 & 32571605 & $5.55 \mathrm{E}-07$ & 9.50 & \\
\hline FLA & qFLAn2-5 & Chr2_33332579 & 2 & 33332579 & $1.84 \mathrm{E}-06$ & 9.33 & \\
\hline FLA & qFLAn6-1 & Chr6_1083411 & 6 & 1083411 & $1.46 \mathrm{E}-07$ & 9.63 & \\
\hline FLA & qFLAn7-2 & Chr7_20475568 & 7 & 20475568 & $2.29 \mathrm{E}-06$ & 9.90 & \\
\hline & & & & & & & \\
\hline
\end{tabular}


Table 2

Significant SNPs associated with flag leaf size related traits in natural population in 2020

\begin{tabular}{|c|c|c|c|c|c|c|c|}
\hline Traits & QTLs & Peak SNPs & Chr. & Position & $P$ value & $R^{2}(\%)$ & known genes \\
\hline $\mathrm{FL}$ & qFLn3-1 & Chr3_7448808 & 3 & 7448808 & 3.94E-06 & 8.87 & \\
\hline $\mathrm{FL}$ & qFLn3-2 & Chr3_8333379 & 3 & 8333379 & 5.01E-06 & 8.77 & \\
\hline $\mathrm{FL}$ & qFLn3-3 & Chr3_8556595 & 3 & 8556595 & $8.66 \mathrm{E}-07$ & 10.00 & \\
\hline FW & $q F W n 1-1$ & Chr1_6294146 & 1 & 6294146 & $6.96 \mathrm{E}-07$ & 10.12 & \\
\hline FW & $q F W n 1-3$ & Chr1_33883944 & 1 & 33883944 & $6.21 \mathrm{E}-07$ & 10.21 & \\
\hline FW & $q F W n 2-1$ & Chr2_30499110 & 2 & 30499110 & $1.60 \mathrm{E}-06$ & 9.50 & \\
\hline FW & qFWn5-2 & Chr5_17982797 & 5 & 17982797 & 7.93E-07 & 10.03 & SNFL 1 \\
\hline FW & $q F W n 6-1$ & Chr6_3907011 & 6 & 3907011 & 7.32E-07 & 10.09 & \\
\hline FW & $q F W n 6-2$ & Chr6_10031161 & 6 & 10031161 & 1.63E-06 & 9.49 & \\
\hline FW & $q F W n 7$ & Chr7_20345187 & 7 & 20345187 & $2.56 \mathrm{E}-06$ & 9.29 & \\
\hline FW & qFWn9-1 & Chr9_16271846 & 9 & 16271846 & $1.20 \mathrm{E}-06$ & 9.72 & \\
\hline FW & $q F W n 9-2$ & Chr9_16890634 & 9 & 16890634 & 4.80E-06 & 8.69 & \\
\hline FW & $q F W n 10-1$ & Chr10_2188025 & 10 & 2188025 & $3.91 \mathrm{E}-06$ & 8.88 & \\
\hline FW & $q F W n 12-1$ & Chr12_478610 & 12 & 478610 & 4.30E-06 & 8.82 & \\
\hline FLA & $q F L A n 1-1$ & Chr1_3318081 & 1 & 3318081 & $5.09 \mathrm{E}-07$ & 10.39 & \\
\hline FLA & $q F L A n 1-2$ & Chr1_33977764 & 1 & 33977764 & $2.05 \mathrm{E}-06$ & 9.34 & OsDET1 \\
\hline FLA & $q F L A n 2-1$ & Chr2_12992454 & 2 & 12992454 & $1.28 \mathrm{E}-06$ & 9.69 & \\
\hline FLA & qFLAn3-1 & Chr3_15386980 & 3 & 15386980 & $1.00 \mathrm{E}-06$ & 10.12 & \\
\hline FLA & qFLAn3-2 & Chr3_16106964 & 3 & 16106964 & 4.33E-07 & 10.51 & \\
\hline FLA & $q F L A n 4$ & Chr4_6025108 & 4 & 6025108 & 3.01E-06 & 9.06 & \\
\hline FLA & $q F L A n 7-1$ & Chr7_5494006 & 7 & 5494006 & $1.50 \mathrm{E}-06$ & 9.57 & \\
\hline FLA & $q F L A n 10-1$ & Chr10_10107835 & 10 & 10107835 & 4.32E-06 & 8.79 & \\
\hline
\end{tabular}

\section{Identification of Pleiotropic QTLs for Flag Leaf Size in Japonica Rice}

In this study, different materials and different analysis methods were used to detect QTLs related to flag leaf size for japonica rice in 2 years. QTLs detected by the two methods and whose physical positions of chromosomes coincided were defined as co-location QTLs (pleiotropic QTLs) (Table 3). In co-location QTL qFL2, qFWr2-3 located in C2_33,142,844-C2_35,004,908 was detected in RILsA in both 2019 and 2020. This region contains the lead SNP Chr2_33,332,579 (qFLAn2-5, qFWn2-2) detected by GWAS and significantly associated with both FW, FLA of japonica rice. The other co-location QTL (pleiotropic QTL) qFL 10 contains linkage analysis QTL qFWr10 located in 
C10_9,054,066 - C10_10,570,732 interval, which was repeatedly detected in 2019 and 2020. According to the physical location of rice chromosome, qFLAn10-1 and $q F W n 10-2$ were both located in $q F W r 10$ interval, and their physical locations are partially coincident.

These 2 pleiotropic QTLs $q F L 2$ and $q F L 10$ were the most important ones that stable expressed through linkage analysis and GWAS in the same interval in this study, indicating that the corresponding interval probably contain the candidate genes of flag leaf size of japonica rice. Thus, further researches will conduct on them.

Table 3

The name and distribution of the co-location QTLs

\begin{tabular}{|c|c|c|c|c|c|c|c|c|c|}
\hline \multirow{2}{*}{$\begin{array}{l}\text { QTL } \\
\text { name }\end{array}$} & \multirow[t]{2}{*}{ Chr. } & \multicolumn{5}{|l|}{ GWAS } & \multicolumn{3}{|l|}{ Linkage mapping } \\
\hline & & $\begin{array}{l}\text { Original } \\
\text { QTL }\end{array}$ & Peak SNP & $\mathrm{P}$ & $R^{2}(\%)$ & $\begin{array}{l}\text { Original } \\
\text { QTL }\end{array}$ & QTL interval & LOD & $R^{2}(\%)$ \\
\hline \multirow[t]{2}{*}{$q F L 2$} & 2 & $q F L A n 2-5$ & Chr2_33332579 & $\begin{array}{l}1.84 \mathrm{E}- \\
06\end{array}$ & 9.33 & \multirow[t]{2}{*}{${ }_{3}^{q F W r 2-}$} & $\begin{array}{l}\text { C2_33142844 - } \\
\text { C2_35004908 }\end{array}$ & 4.65 & 9.46 \\
\hline & & $q F W n 2-2$ & Chr2_33332579 & $\begin{array}{l}3.18 \mathrm{E}- \\
08\end{array}$ & 12.45 & & $\begin{array}{l}\text { C2_33142844 - } \\
\text { C2_35004908 }\end{array}$ & 2.52 & 7.27 \\
\hline \multirow[t]{2}{*}{ qFL 10} & 10 & $\underset{1}{q F L A n 10-}$ & Chr10_10107835 & $\begin{array}{l}4.32 \mathrm{E}- \\
06\end{array}$ & 8.79 & \multirow[t]{2}{*}{ qFWr10 } & $\begin{array}{l}\text { C10_9054066 - } \\
\text { C10_10570732 }\end{array}$ & 2.59 & 8.15 \\
\hline & & ${ }_{2}^{q F W n 10-}$ & Chr10_10230100 & $\begin{array}{l}1.67 \mathrm{E}- \\
06\end{array}$ & 10.32 & & $\begin{array}{l}\text { C10_9054066 - } \\
\text { C10_10570732 }\end{array}$ & 2.86 & 11.26 \\
\hline
\end{tabular}

\section{Candidate Gene Screening and Haplotype Analysis}

The P of lead SNP Chr2_33, 332, 579 of $q F W n 2-2$ in $q F L 2$ is the smallest, which is 3.18E-08. Considering that the LD of the whole genome is $109 \mathrm{~kb}$ (Fig. 3A), we selected $109 \mathrm{~kb}$ upstream and downstream of this SNP as the target interval to screen candidate genes. There are 37 genes in the target region, including 22 function annotated genes, 5 expression proteins with unknown function, 5 hypothetical proteins and 5 retrotransposon proteins (Table S5).We used SNPs with nonsynonymous mutations in exons to analyze the haplotypes of these genes and found that there were 2 functional annotation genes LOC_Os02g54254, LOC_Os02g54550 had significant differences in FW among different haplotypes, and the differences in 2019 and 2020 were basically the same (Fig. 4). Table 4,showed that 2 haplotypes of LOC_Os02g54254 (G/A) and LOC_Os02g54550(C/T)had significant differences in FW. qFL 10,apleiotropic QTL either was composed of stable QTLs detected by linkage analysis and significant SNPs detected by GWAS. There is an overlapping interval between the two ways results, that is, the $10.12 \mathrm{Mb}-10.22 \mathrm{Mb}$ interval of chromosome 10 (overlapping interval of $q F L A n 10-1[q F W n 10-2$ ) to be the target interval (Fig. 3B, C). Fifteen genes exist in the target region, including 5 function annotated genes, 4 expression proteins with unknown function and 6 retrotransposon proteins (Table S6, Fig. 3D). SNPs with nonsynonymous mutations in exons were used to analyze the haplotypes of the genes, and 3 functional annotation genes

LOC_Os10g20160,LOC_Os10g20240 and LOC_Os10g20260 were found to have significant differences in FW among different haplotypes, and the differences in 2019 and 2020 were basically the same (Fig. 4). Haplotype analysis revealed that significant differences for FW were observed between hap1 (TGT), hap2 (TAT) and hap3 (CGT) in LOC_Os10g20160. LOC_Os10g20240 and LOC_Os10g20260 both had 2 haplotypes as hap1 (GA), hap2(AC) and hap1 (CCC), hap2(CCG) which showed significant difference for FW. 
Table 4

Candidate gene haplotypes and the number of varieties corresponding to each haplotype

\begin{tabular}{|llll|}
\hline Gene & Hap1/Number & Hap2/Number & Hap3/Number \\
\hline LOC_Os02g54254 & $\mathrm{G} / 274$ & $\mathrm{~A} / 17$ & \\
\hline LOC_Os02g54550 & $\mathrm{C} / 275$ & $\mathrm{~T} / 14$ & \\
\hline LOC_Os10g20160 & $\mathrm{TGT} / 217$ & $\mathrm{TAT} / 30$ & CGT/18 \\
\hline LOC_Os10g20240 & $\mathrm{GA} / 279$ & $\mathrm{AC} / 11$ & \\
\hline LOC_Os10g20260 & $\mathrm{CCC} / 255$ & $\mathrm{CCG} / 17$ & \\
\hline
\end{tabular}

\section{Identification of candidate genes based on qRT-PCR}

According to the results of haplotype analysis, 5 candidate genes were analyzed by qRT-PCR using 4 RILs parents (K131, HDB, WD20342, Caidao) as templates in 4 growth periods from the flag leaf begin to drew out to fully extended. The candidate genes and quantitative primers are shown in Table S1. Expressions of LOC_Os02g54254, LOC_Os02g54550, LOC_Os10g20240, LOC_Os10g20260, had no obvious regularity and did not increase significantly in a certain period (Fig. 5). On the other hand, LOC_Os10g20160 presented different expression type, and the expression level of the RILs parents in 4 periods was significantly higher than that of other genes. LOC_Os10g20160 (SD-RLK-45) belongs to S-domain receptor-like kinases (SD-RLK) family shows preferential in leaf, shoot and seeds (Aya et al. 2011). Thus, SD-RLK-45 is probably the candidate gene of $q F L 10$.

\section{Discussion}

Leaf type improvement is one of the important ways to increase rice yield (Dastan et al. 2020). By rationally controlling leaf type traits could enhance lodging resistance, photosynthetic utilization rate, and what's more, increase yield per plant (Ya et al. 2015; Clerget et al. 2016). Japonica rice has better cooking and eating quality due to higher amylose content, which cultivated and consumed in East Asia as the major variety (https://en.wikipedia. org/wiki/Japonica_rice), and is obviously worth to conduct researches on the genetic variation of leaf type (size) of japonica rice. Although some genes or QTLs regulating flag leaf size were identified by classical mapping and reverse genetics, the number of studies on leaf genetic variation about japonica rice is still relatively small (Jiang et al. 2010; Yang et al. 2015; Hu et al. 2012). In this study, three japonica rice populations were used for linkage mapping and GWAS in 2 years, and 64 leaf shape QTLs were mapped, some of which were overlapped or similar to that in previous studies, and some were novel QTLs. Interval of $q F L r 6-2$ and $q F L A r 3-1$ detected by linkage mapping overlapped with $q F L L 6$ and $q L A 3-1$ (Shen et al, 2011) regulating leaf length and leaf area respectively. $q F L L 1.2$ mapped by Zhang et al, (2015b) using an RIL population and resequencing genetic map was found to be located within $q F L r 1$ possessing same function. The recognized narrow leaf gene $N A L 1$ ( Li et al, 2019a) on chromosome 4 of rice was found to be located within qFLAr4-4 in this study. qFWn7located in the same interval with OsFLW7 identified by Xu et al (2017). Known genes OsBAK1 (Li et al, 2009), SNFL 1 (He et al, 2018) and OsDET1 (Zang et al, 2016), were found to be located within or nearby the LD interval of $q F W n 8, q F W n 5-2, q F L A n 1-2$ in this study. Among these genes, OsBAK1 controlling leaf angle and length by regulating brassinosteroid (BRs), and has been observed significantly reduce plant height. The study of SNFL1 mutant indicated that the length of epidermal cells and the number of longitudinal veins in flag leaves decreased remarkably. OsDET1 has been proved to regulate ABA signal transduction and play an important role in maintaining rice growth and plant type development. 
Through 2 years of GWAS and linkage analysis, we found pleiotropic and stable QTLs $q F L 2$ and $q F L 10$, representing stable genetic effects. In view of the genome-wide LD decay of GWAS, we selected $218 \mathrm{~kb}$ upstream and downstream to screen candidate genes, and also served as the overlap region of linkage analysis and GWAS (Li et al. 2019b). Based on haplotype analysis, we obtained 5 candidate genes: LOC_Os02g54254 (OsLKR/SDH), LOC_Os02g54550 (OsFBX63), LOC_Os10g20240 (OsKNOLLE), LOC_Os10g20260 (CSIF7), LOC_Os10g20160 (SD$R L K-45)$. OsLKR/SDH was proved to be a bifunctional lysine degrading enzyme (Takaiwa et al. 2010), OsFBX63 is a F-box family gene, the function of which hasn't been studied. Syntaxin-related protein OsKNOLLE probably play an important role in regulating abiotic stress resistance (Wang et al. 2011). CSIF7 was confirmed as a Cellulose Synthase family gene (Julian et al. 2015). SD-RLK-45 belongs to $S D-R L K$ family and supposed to be a novel functional gene. Characteristics of expression during leaf development period make $S D-R L K-45$ the most likely candidate gene for $q F L 10$.

Plant receptor-like protein kinases ( $R L K S$ ) comprise one of the largest and most diverse superfamily of plant proteins with 610 and 1131 members in the Arabidopsis and rice genomes, respectively (Zou et al. 2015). The RLKs gene superfamily played fundamental roles in hormone perception, developmental regulation, innate immunity, adaptation to abiotic stresses, and quantitative yield components (Melissa et al. 2009; Herder et al. 2012, pan et al. 2019). S-domain RLKs (SD-RLKS) belongs to a subfamily of $R L K s$, with 147 members in rice. (Morillo et al. 2006) Recent studies on rice have confirmed that OSSRK1 regulates leaf width by promoting cell division in the leaf primordium and OSSRK1-overexpression plants exhibited enhancing ABA sensitivity and salt tolerance compared with wild type (Zhou et al. 2020).

In the present study, there were significantly differences in flag leaf width of the haplotypes of 5 candidate genes OsLKR/SDH, OsFBX63, OsKNOLLE, CSIF7 and SD-RLK-45. Only the expression of SD-RLK-45 showed excellent characteristics during flag leaf development, and it's most likely to be the candidate gene of $q F L 10$. As a novel gene that has not been systematically studied, additional data are needed to verify the function of $S D-R L K-45$ in controlling flag leaf width or size. The overexpression, construction of CRISPR-Cas 9 and omics experiments will be the focus of our future studies.

\section{Conclusions}

Two RILs and 295 japonica rice varieties were collected to identify the flag leaf size phenotypic. Two pleiotropic QTLs qFL2, qFL 10 consisted of overlapping QTLs mapped by linkage analysis and GWAS were identified. Based on LD decay distance and pleiotropic interval overlapping, 2 intervals of 218-kb and 100-kb were selected for candidate gene screening. LOC_Os02g54254, LOC_Os02g54550, LOC_Os10g20160, LOC_Os10g20240, LOC_Os10g20260 were identified by haplotype analysis as candidate genes, and qRT-PCR showed LOC_Os10g20160 probably to be a novel functional gene contributing flag leaf size by regulating flag leaf width of japonica rice. The results provide resources for leaf type improvement breeding.

\section{Declarations}

\section{Funding}

This research was supported by the Heilongjiang Provincial government Postdoctoral Foundation of China (LBHZ16188), the Natural Science Foundation Joint Guide Project of Heilongjiang (LH2019C035) and the ProvinceAcademy Science and Technology Cooperation Project of Heilongjiang (YS20B05). Application R \& D Project of Heilongjiang Academy of Agricultural Science (2021YYYF037). 


\section{Author details}

1 Heilongjiang Academy of Agricultural Sciences, Institute of Crops Tillage and Cultivation. Harbin 150030, China. 2 Key Laboratory of Germplasm Enhancement, Physiology and Ecology of Food Crops in Cold Region, Ministry of Education, Northeast Agricultural University, Harbin 150030, China.

\section{Ethical Statement}

The authors of this paper declare that we have no conflict of interest. Euphytica is the only journal we submitted. The submitted work is original and haven't been published elsewhere in any form or language. This article does not contain any studies with animals performed by any of the authors. Informed consent was obtained from all individual participants included in the study.

\section{References}

1. Adachi M, Hasegawa, Toshihiro F, Hiroshi et al (2014) Soil and water warming accelerates phenology and downregulation of leaf photosynthesis of rice plants grown under free-air co2 enrichment (face). Plant \& Cell Physiology

2. Aya K, Suzuki G, Suwabe K, Hobo T, Takahashi H, Shiono K, et al (2011) Comprehensive network analysis of anther-expressed genes in rice by the combination of 33 laser microdissection and 143 spatiotemporal microarrays. PLoS ONE 6(10):e26162

3. Barboza LGA, Vethaak AD, Lavorante BR, Lundebye AK, Guilhermino L (2018) Marine microplastic debris: An emerging issue for food security, food safety and human health. Mar Pollut Bull 133:336-348

4. Bradbury PJ, Zhang Z, Kroon DE, Casstevens TM, Ramdoss Y, Buckler ES (2007) TASSEL: software for association mapping of complex traits in diverse samples. Bioinformatics 23:2633-2635

5. Clerget B, Domingo AJ, Layaoen, \& HL, et al (2016) Leaf emergence, tillering, plant growth, and yield in response to plant density in a high-yielding aerobic rice crop. FIELD CROP RES, 2016,199(-), 52-64

6. Chen M, Luo J, Shao G, Wei X, Tang S, Sheng Z, Song J, Hu P (2012) Fine mapping of a major QTL for fag leaf width in rice, $q F L W 4$, which might be caused by alternative splicing of NAL 1. Plant Cell Rep 31:863-872.1

7. Dastan S, Ghareyazie B, Silva J (2020) Selection of ideotype to increase yield potential of gm and non-gm rice cultivars. Plant Sci 279:110519

8. Famoso AN, Zhao K, Clark RT, Tung CW, Wright MH, Bustamante C, et al (2011) Genetic architecture of aluminum tolerance in rice (oryza sativa) determined through genome-wide association analysis and qtl mapping. Plos Genetics 7(8):747-757

9. Farooq M, Tagle AG, Santos RE, Ebron LA, Fujita D, Kobayashi N (2010) Quantitative trait loci mapping for leaf length and leaf width in rice cv. IR64 derived lines. J Integr Plant Biol 52:578-584

10. Fujino K., Matsuda Y., Ozawa K., Nishimura T., Koshiba T., W.Fraaije M., and Sekiguchi H., (2008), Narrow leaf 7 controls leaf shape mediated by auxin in rice, Mol. Genet. Genom., 279: 499-507.

11. Giuliani R, Koteyeva N, Voznesenskaya E, Evans MA, Cousins AB, Edwards GE (2013) Coordination of leaf photosynthesis, transpiration, and structural traits in rice and wild relatives (Genus Oryza). Plant Physiol 162:1632-1651

12. Gu J, Yin X, Tjeerd-Jan S, Wang H, Struik PC. (2012). Physiological basis of genetic variation in leaf photosynthesis among rice (oryza sativa I.) introgression lines under drought and well-watered conditions. Journal of Experimental Botany(14), 5137 
13. He W, Shunsuke, Adachi R, \& F., et al (2017) Leaf photosynthetic rate and mesophyll cell anatomy changes during ontogenesis in backcrossed indicaxjaponica rice inbred lines. Photosynth Res 134(1):1-12

14. He PL, Wang XW, Zhang XB, Tian JiangYD, Zhang WJ, Li XQ, Sun YY, Xie Y, Ni J, He JL G.H., and Sang XC, (2018), Short and narrow flag leaf1, a GATA zinc finger domain-containing protein, regulates flag leaf size in rice (Oryza sativa L.)

15. Herder GD, Yoshida S, Antolin-Llovera M, Ried MK, Parniske M (2012) Lotus japonicus e3 ligase seven in absentia4 destabilizes the symbiosis receptor-like kinase symrk and negatively regulates rhizobial infection. Plant Cell 24(4):1691-1707

16. Hoang GT, Gantet P, Nguyen KH, Phung N, Xuan HP (2019) Genome-wide association mapping of leaf mass traits in a vietnamese rice landrace panel. PLoS ONE 14(7):e0219274

17. Hu W, Zhang H, Jiang J, Wang Y, Sun D, Wang X, Hong D (2012) Discovery of a germplasm with large flag leaf angle and its genetic analysis as well as QTL mapping in japonica rice. Chinese Journal of Rice Science 26(1):34-42

18. Jiang SK, Zhang XJ, Zheng-Jin XU, Wen-Fu C (2010) Comparison between QTLs for chlorophyll content and genes controlling chlorophyll biosynthesis and degradation in japonica rice. Acta agronomica sinica 36(3):376384

19. Jiang D, Fang JJ, Lou LM, Zhao JF, Yuan SJ, Yin L, Sun W, Peng LX, Guo BT, Li XY (2015) Characterization of a null allelic mutant of the rice NAL 1 gene reveals its role in regulating cell division. PLoS One 10(2):e0118169

20. Julian G, Schwerdt, Katrin MacKenzi, Frank et al (2015) Evolutionary dynamics of the cellulose synthase gene superfamily in grasses. Plant Physiology, 168(3)

21. Julio R, Albert FM, Sánchez-DelBarrio J, Carlos, Sara GR, Pablo L, Ramos-Onsins SE, et al (2017) Dnasp 6: dna sequence polymorphism analysis of large data sets. Molecular Biology \& Evolution (12), 12

22. Li A, Yz, B, Fang, L. B, Qian, C. B, \& Jq, A. (2019a). Narrow leaf 1 (NAL1) regulates leaf shape by affecting cell expansion in rice (oryza sativa I.). Biochemical and Biophysical Research Communications, 516 (3), $957-962$.

23. Li D, Wang, L, Wang, M., Xu, Y., Luo, W., \& Liu, Y., et al. (2009). Engineering osbak1 gene as a molecular tool to improve rice architecture for high yield. Plant Biotechnology Journal, 7(8), 791-806.

24. Li N, Zheng, H., Cui, J., Wang, J, \& D Zou. (2019b). Genome-wide association study and candidate gene analysis of alkalinity tolerance in japonica rice germplasm at the seedling stage. Rice, 12(1).

25. Li X, Zheng H, Wu W, Liu H, Zhao H (2020) QTL mapping and candidate gene analysis for alkali tolerance in japonica rice at the bud stage based on linkage mapping and genome-wide association study. Rice, 13(1)

26. Livak KJ, Schmittgen TD (2001) Analysis of relative gene expression data using real-time quantitative PCR and the $2^{-\Delta \Delta C T}$ method. Methods 25:402-408

27. Meng L, Li H, Zhang L, Wang J (2015) Qtl icimapping: integrated software for genetic linkage map construction and quantitative trait locus mapping in biparental populations - sciencedirect. The Crop Journal 3(3):269-283

28. Melissa D. Lehti-Shiu C, Zou K, Hanada, Shin-Han S (2009) Evolutionary history and stress regulation of plant receptor-like kinase/pelle genes. Plant Physiol 150(1):12-26

29. Morillo SA, Tax FE (2006) Functional analysis of receptor-like kinases in monocots and dicots. Curr Opin Plant Biol 9(5):460-469

30. Pan J, Li Z, Wang Q, Yang L, Yao F, Liu W (2019) An s-domain receptor-like kinase, osesg1, regulates early crown root development and drought resistance in rice. Plant Science, 290 
31. Pane TC, Supriyono Y, Novita D. (2021). Supporting food security with rice farming insurance: the farmers' perceptions (case study in cinta damai village, percut sei tuan subdistrict, deli serdang district). IOP Conference Series: Earth and Environmental Science, 782(2), 022044 (8pp)

32. Rahman MA, Haque ME, Sikdar B, Islam MA, Matin MN (2013) Correlation analysis of flag leaf with yield in several rice cultivars. Journal of Life Earth Science 8:49-54

33. Shen B, YU W D, DU JH et al (2011) Validation and dissection of quantitative trait loci for leaf traits in interval RM4923-RM402 on the short arm of rice chromosome. Genet 90(1):39-44

34. Tang X, Gong R, Sun, \& W., et al (2018) Genetic dissection and validation of candidate genes for flag leaf size in rice (oryza sativa I.). THEORETICAL AND APPLIED GENETICS

35. Timmer CP (2014) Food security in Asia and the pacific: the rapidly changing role of rice. Asia \& the Pacific Policy Studies

36. Takaiwa $F$ (2010) Differences in transcriptional regulatory mechanisms functioning for free lysine content and seed storage protein accumulation in rice grain. Plant Cell Physiology 51(12):1964-1974

37. Turner SD (2014) Qqman: an r package for visualizing gwas results using q-q and manhattan plots. Biorxiv

38. Wang F, Zhu C (2011) Heterologous expression of a rice syntaxin-related protein KNOLLE gene (OsKNOLLE) in yeast and its functional analysis in the role of abiotic stress. genetics 33(11):1251-1257

39. Xu J., Wang L., Wang Y.X., Zeng D.L., Zhou M.Y., Fu X., Ye W.J., Hu J., Zhu L., Ren D.Y., Gao Z.Y., Dong G.J., Guo L.B., Zhang G.H., and Qian Q., (2017), Reduction of OsFLW7 expression enhanced leaf area and grain production in rice expression enhanced leaf area and grain production in rice, Science Bulletin, 62(24): 1631-1633.

40. Ya-Jie, H. U., Cao, W. W., Qian, H. J., Xing, Z. P., Zhang, H. C., \& Dai, Q. G., et al. (2015). Effect of planting density of mechanically transplanted pot seedlings on yield, plant type and lodging resistance in rice with different panicle types. ACTA AGRONOMICA SINICA.

41. Yang W, Guo Z, Huang C, Wang K, Jiang N, Feng H, Chen G, Liu Q, Xiong L (2015) Genome-wide association study of rice (Oryza sativa L.) leaf traits with a high-throughput leaf scorer. J Exp Bot 66:5605-5615

42. Zang G, Zou H, Zhang Y, Xiang Z, Huang J, Luo L et al (2016) OsDET1 modulates the aba signaling pathway and aba biosynthesis in rice. Plant Physiology, 1259

43. Zhang G, Li S, Wang L, Ye W, Zeng D, Rao Y, Peng Y, Hu J, Yang Y, Xu J, Ren D, Gao Z, Zhu L, Dong G, Hu X, Yan M, Guo L, Li C, Qian Q (2014) LSCHL 4 from Japonica cultivar, which is allelic to NAL 1, increases yield of Indica super rice 93 - 11. Mol Plant 7:1350-1364

44. Zhang B, Ye W, Ren D, Tian P, Peng Y, Gao Y, Ruan B, Wang L, Zhang G, Guo L, Qian Q, Gao Z (2015a) Genetic analysis of flag leaf size and candidate genes determination of a major QTL for flag leaf width in rice. Rice (New York NY) 8(1):39. https://doi.org/10.1186/s12284-014-0039-9

45. Zhang B, Ye W, Ren D, Tian P, Peng Y, Gao Y et al (2015b) Genetic analysis of flag leaf size and candidate genes determination of a major QTL for flag leaf width in rice. Rice, 8(1)

46. Zhou, Jinjun JU, Peina ZHANG, Fang et al (2020) Ossrk1, an atypical s-receptor-like kinase positively regulates leaf width and salt tolerance in rice. Rice Sci 27(02):57-66

47. Zhu X, Song Q, Ort DR (2012) Elements of a dynamic systems model of canopy photosynthesis. Curr Opin Plant Biol 15:237-244

48. Zhu C, Zhu J, Cao J, Jiang Q, Liu G, Ziska LH (2014) Biochemical and molecular characteristics of leaf photosynthesis and relative seed yield of two contrasting rice cultivars in response to elevated [co2]. J Exp Bot 65(20):6049 
49. Zou X, Qin Z, Zhang C, Liu B, Liu J, Zhang C et al (2015) Over-expression of an s-domain receptor-like kinase extracellular domain improves panicle architecture and grain yield in rice. Journal of Experimental Botany (22), 7197-7209

\section{Figures}
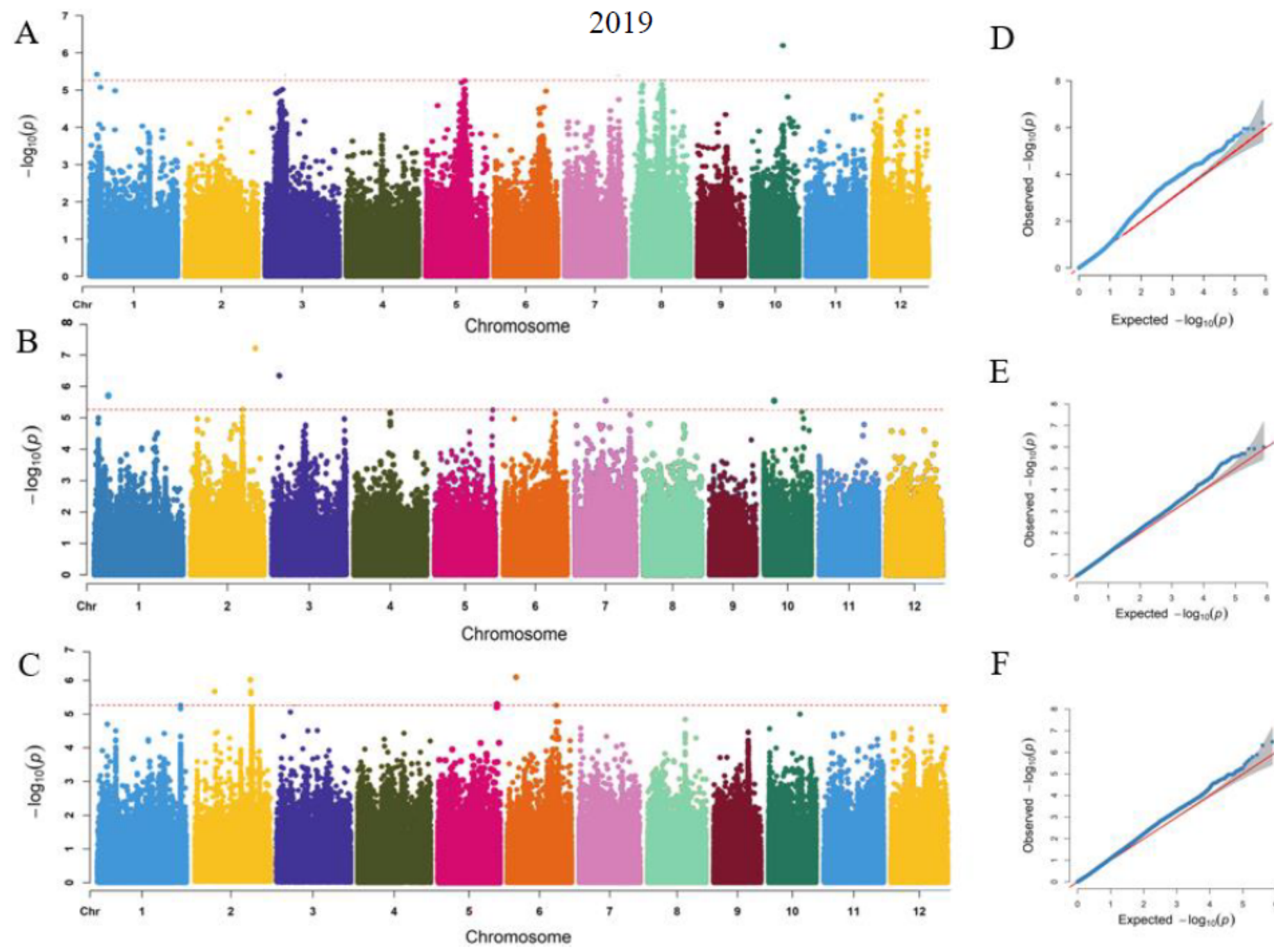

$\mathrm{E}$

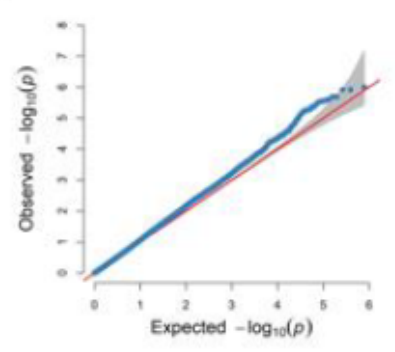

$\mathrm{F}$

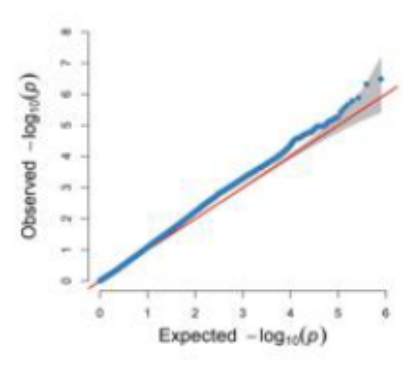

Figure 1

Manhattan plots and quantile-quantile (Q-Q) plots of genome-wide association studies for FL, FW and FLA in 2019. A, Manhattan plot for FL. B, Manhattan plot for FW. C, Manhattan plot for FLA. D, Q-Q plot for FL. E, Q-Q plot for FW. F, Q-Q plot for FLA 

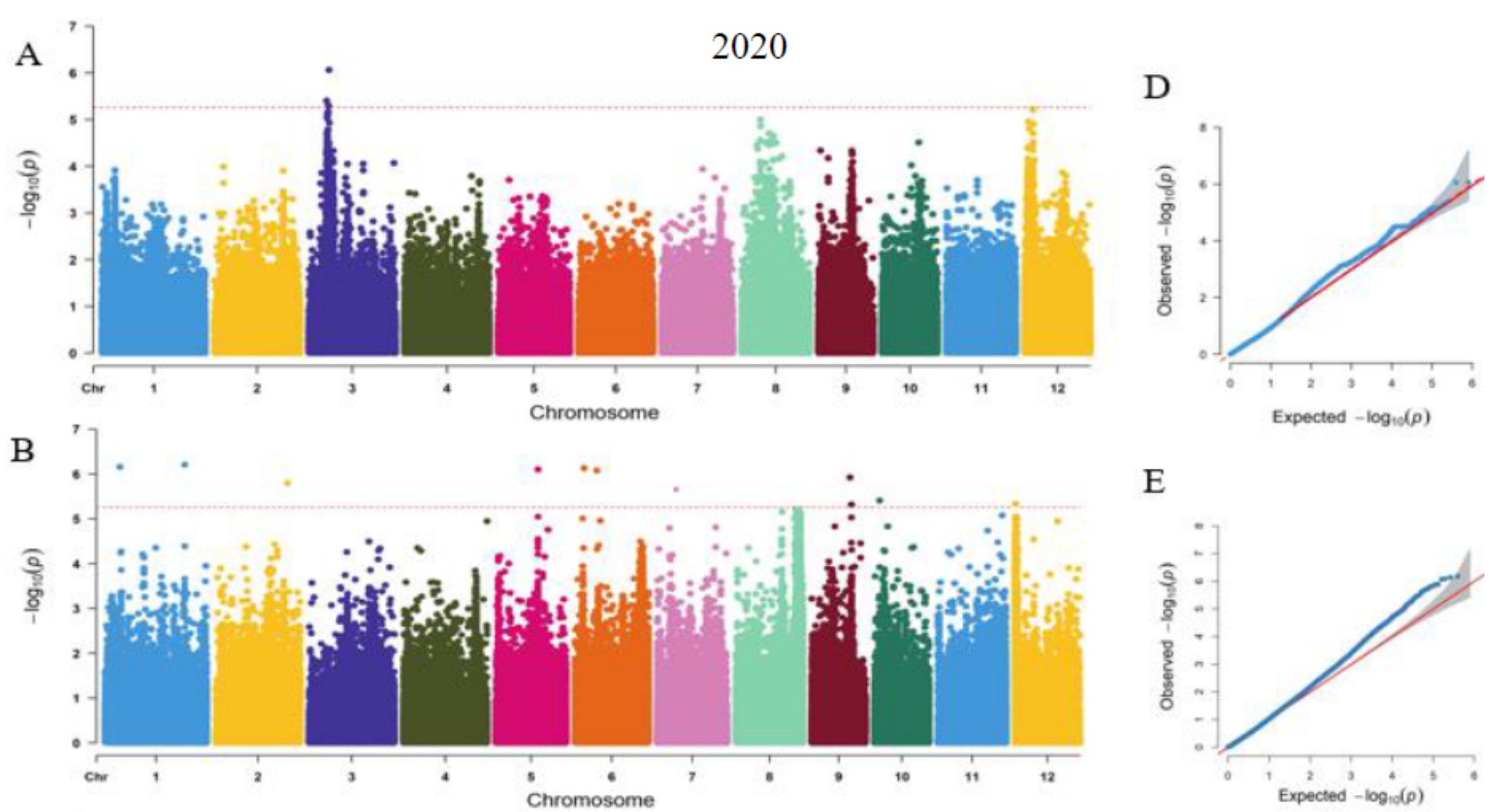

E
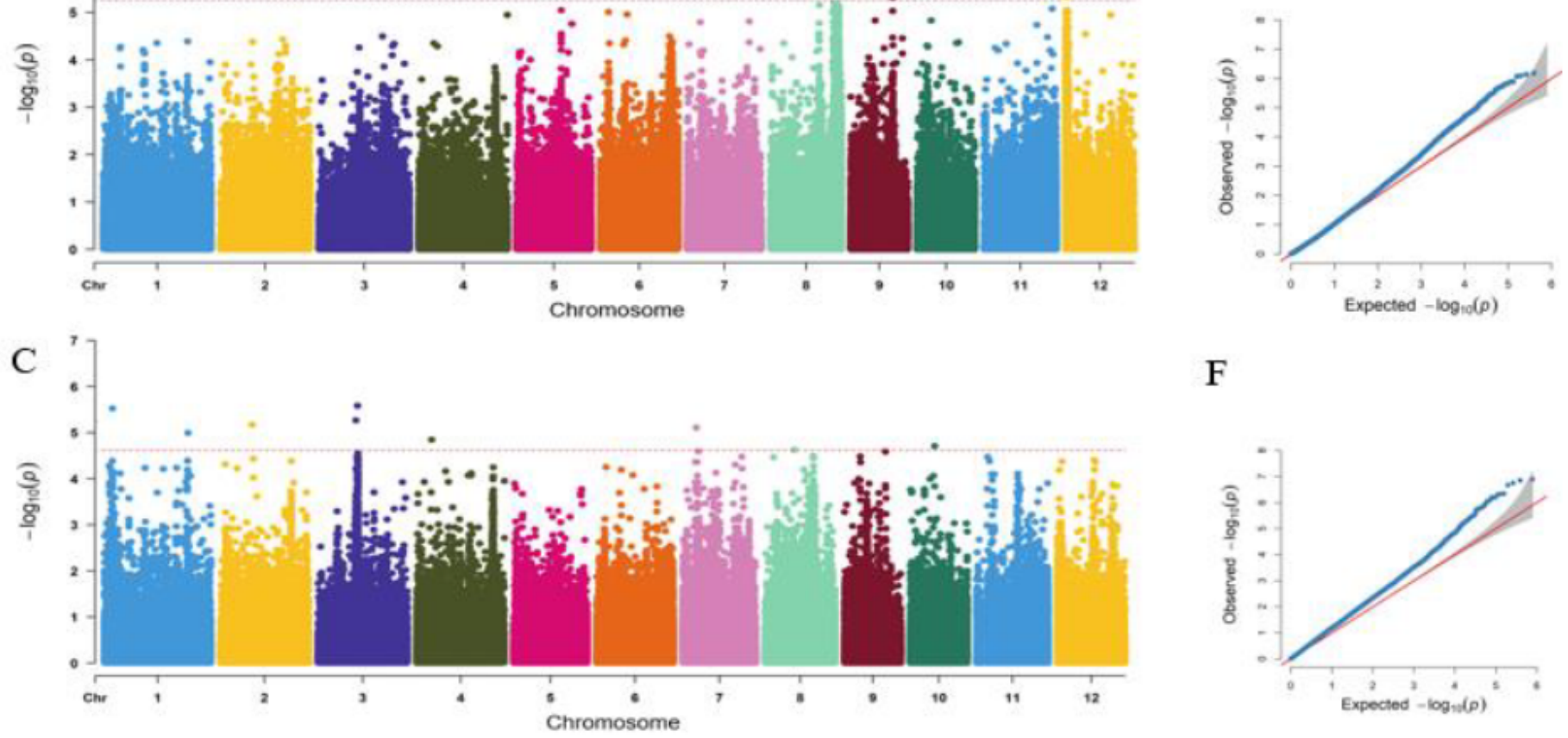

F

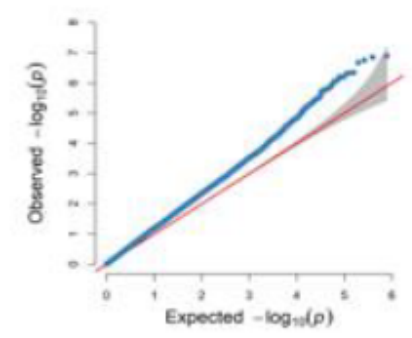

\section{Figure 2}

Manhattan plots and quantile-quantile (Q-Q) plots of genome-wide association studies for FL, FW and FLA in 2020. A, Manhattan plot for FL. B, Manhattan plot for FW. C, Manhattan plot for FLA. D, Q-Q plot for FL. E, Q-Q plot for FW. F, Q-Q plot for FLA 


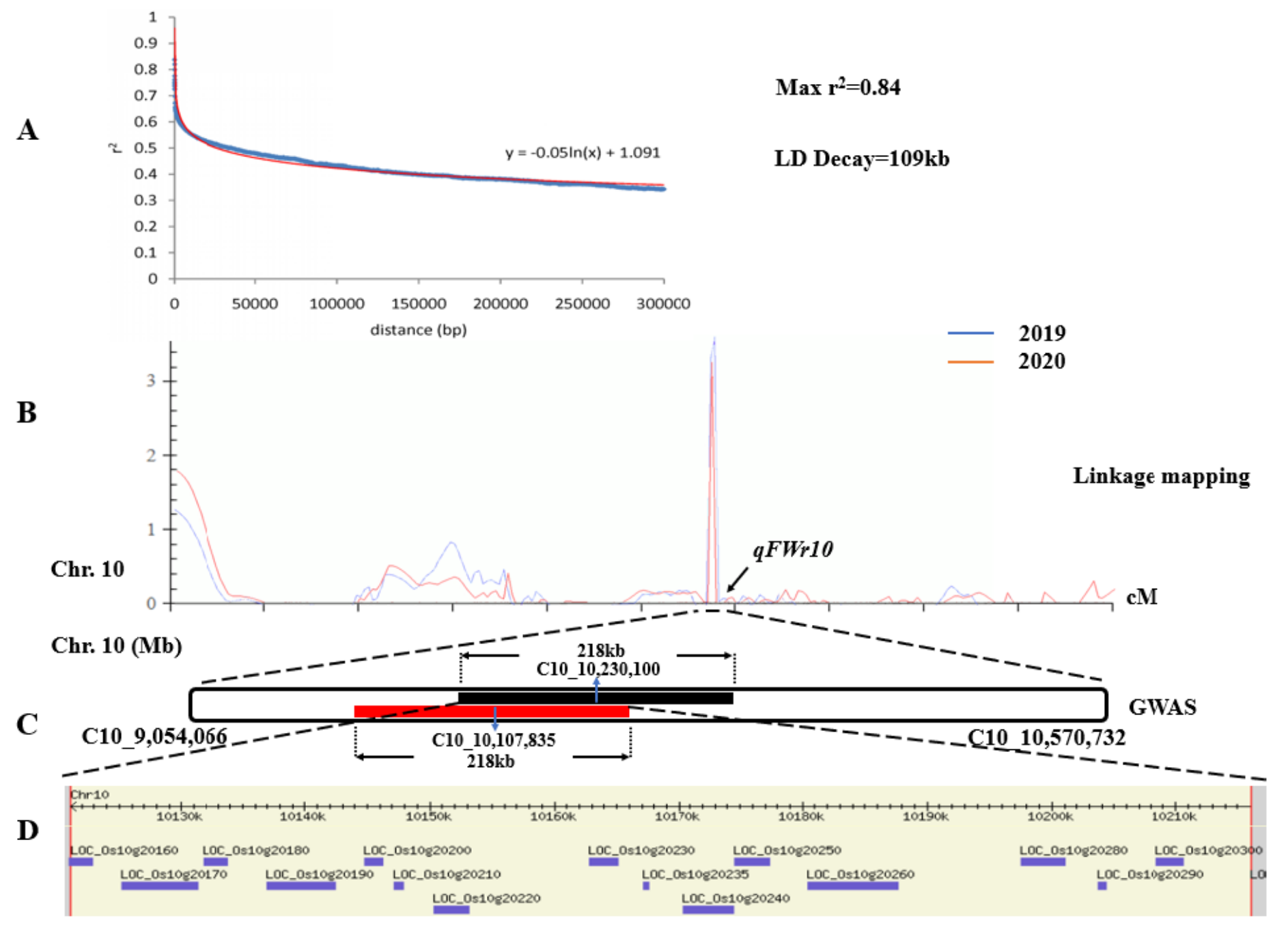

\section{Figure 3}

Identification of candidate genes by linkage mapping and GWAS. A LD decay of the whole genome in 295 japonica rice varieties. When $\mathrm{r} 2$ decays to the half, the corresponding physical distance $(109 \mathrm{~kb})$ is recorded as the LD attenuation distance of the whole genome. B qFWr10 located in C10_9,054,066 - C10_10,570,732 interval, which was repeatedly detected in 2019 and 2020. C Overlapping physical location of the lead SNP (C10_10,107,835, C10_10,230,100) on chromosome 10 detected by GWAS. D 15 genes in the $218 \mathrm{~kb}$ region. 

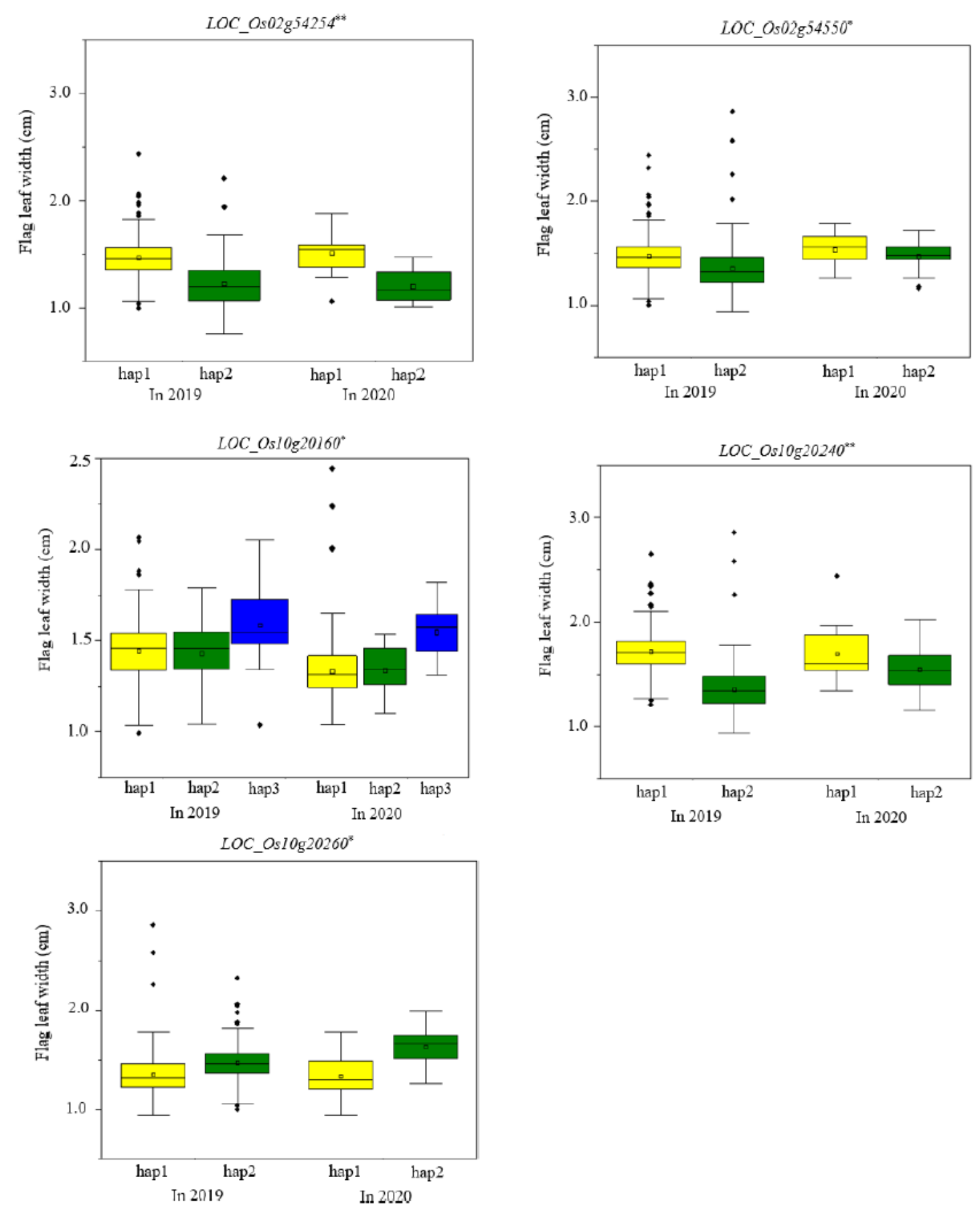

Figure 4

Boxplot for Flag leaf width based on the haplotypes (green, yellow blue indicate the phenotypic result for hap1, hap2 and hap3, respectively (The *and ** suggested significance of ANOVA at $\mathrm{P}<0.05$ and $\mathrm{P}<0.01$, respectively) 

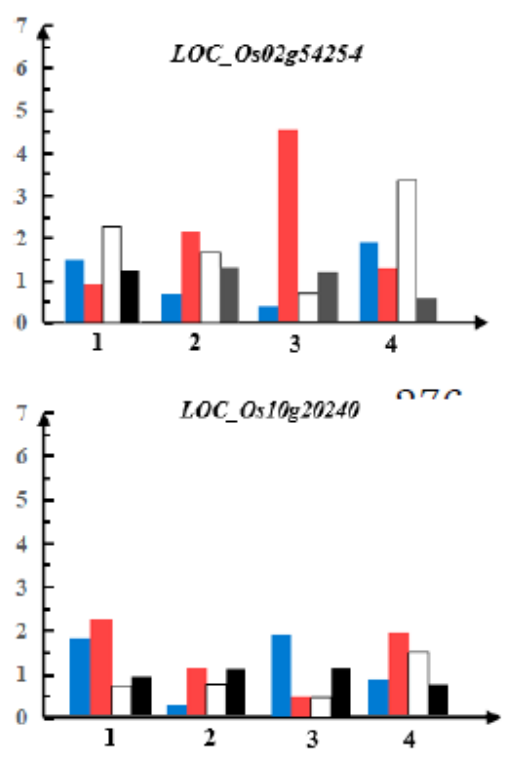
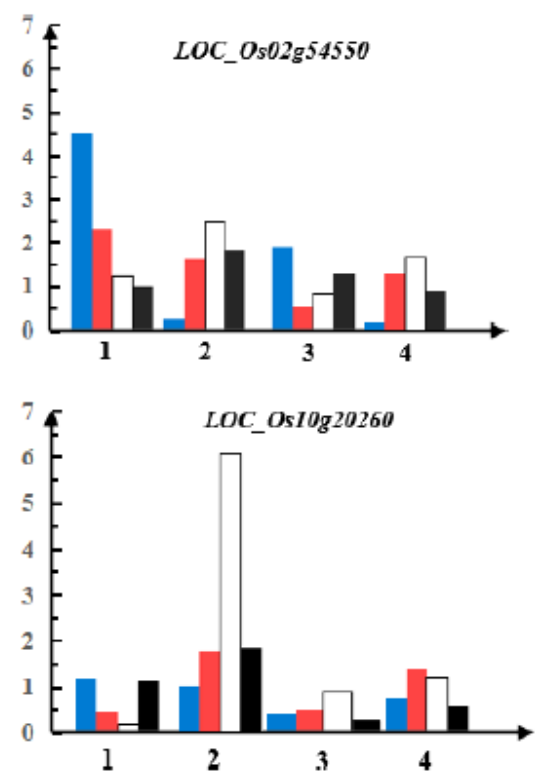
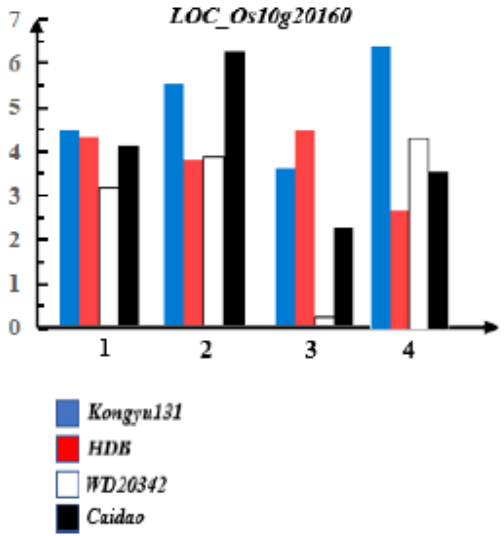

Figure 5

Expression patterns of 5 genes in 4 growth periods

\section{Supplementary Files}

This is a list of supplementary files associated with this preprint. Click to download.

- SupplementaryFigS1GeneticlinkagemapofRILsA.png

- SupplementaryFigS2GeneticlinkagemapofRILsB.png

- SupplementaryTables.xlsx 